\title{
Relationship between Cognitive Learning Psychological Classification and Neural Network Design Elements
}

\author{
Xing Yang, ${ }^{1}$ Tingjun Yong $\mathbb{C D}^{1}{ }^{1}$ Meihua Li, ${ }^{1}$ Wenying Wang, ${ }^{2}$ Huichun Xie, ${ }^{2}$ and Jinping Du ${ }^{3}$ \\ ${ }^{1}$ Department of Education, Qinghai Normal University, Xining 810000, China \\ ${ }^{2}$ School of Life Sciences, Qinghai Normal University, Xining 810000, China \\ ${ }^{3}$ Weihai Gao Experimental Primary School, Weihai 264200, China
}

Correspondence should be addressed to Tingjun Yong; 2018235@qhnu.edu.cn

Received 28 November 2020; Revised 6 January 2021; Accepted 18 February 2021; Published 27 February 2021

Academic Editor: Wei Wang

Copyright (c) 2021 Xing Yang et al. This is an open access article distributed under the Creative Commons Attribution License, which permits unrestricted use, distribution, and reproduction in any medium, provided the original work is properly cited.

\begin{abstract}
This article first analyzes the research background of the design elements of cognitive psychology and neural networks at home and abroad, roughly understands the research status and research background of these two courses at home and abroad, and discusses the application of cognitive psychology to neural networks. The design method has not yet formed a systematic theoretical system. Then, a systematic theoretical analysis of the research in this article is carried out to analyze the relationship between the various characteristics of cognitive psychology and the design elements of the neural network, and it uses these relationships to guide the design practice. Second, it analyzes the relationship between the influence and interaction of cognitive psychology on neural network design and connects cognitive psychology with neural network design. Finally, according to the theoretical analysis and research of the system, the application of cognitive psychology in neural network design, design practice, and the relationship between the two are systematically reviewed. Through the exploratory research on cognitive psychology in neural network design, we can see that the combination of neural network design and psychology, art aesthetics, and other crossdisciplinary and multidisciplinary research is necessary, which can promote the scientific and technological progress of neural network design in the context of the information age and the improvement of public mental health. Under the background of the era in which the neural network design becomes the link between people's emotions and culture, we must fully understand the essential role of each element in neural network design and build a design concept based on cognitive psychology and emotional experience. It is hoped that the content of this topic can provide a certain reference value for the future development of neural network design and cognitive psychology and clarify the new development direction.
\end{abstract}

\section{Introduction}

Today, in the development of psychology, cognitive psychology is the main direction of research. The difference between cognitive psychology and other disciplines is that cognitive psychology is not to explore people's various projections by studying the phenomena that people manifest on the surface but to study the process of information transmitted by objective things to people's brains [1]. Among the many branches of psychology, cognitive psychology studies the complex thinking patterns of people and the process of psychological activity that combines the storage and extraction of information in memory with new knowledge information to produce its own empirical knowledge information. All this constitutes the essential difference between humans and animals. The entire cognitive psychological process greatly affects the behaviors people take in the process of obtaining information. However, compared to other "hard" sciences like physics, which has measurable body stimuli and measures responses to stimuli, the research process of cognitive psychology is obviously more unknown and uncertain [2]. However, cognitive psychology is indeed the core area of many scientific studies. In the past research on information acquisition behavior, most of the research results are from the perspective of external environment and scenarios to analyze information acquisition behavior. In the field of scientific research, nowadays, we have learned more from the 
perspective of people's fundamental needs, people-oriented, and the psychological process of internal information cognition to understand the implementation of the entire information acquisition behavior more fully.

The main figures abroad on cognitive learning models and learning mechanisms are Bzdok, Danilo, and Andreas Meyer-Lindenberg. They proposed a multimedia learning cognitive model combining Baddeley's working memory model, Paivio's double-coding theory, and Sweller's cognitive load theory [3]. On the basis of Mayer's multimedia learning model, Wen, Guihua, and others proposed a comprehensive model of multimedia learning effect, which summarizes the process of multimedia learning into four types of elements: multimedia information input, cognitive processing, learning motivation, and knowledge and learning, among which learning motivation elements included learning style and cognitive participation [4]. American psychologist Kasabov puts people's response factors as a function of variables. This theory also greatly promotes the development speed of cognitive psychology. This subject is gaining more and more attention in society [5]. Kanchanatawan believes that cognition does not occur independently of the human environment, psychological activities, and behavior but is based on various behavioral activities that people engage in. John and Keane of the United States carried out detailed analysis and systematic conclusions on cognitive psychology and further studied people's psychological, physiological, memory, feeling, and other factors. We can see that the research should be more systematic and detailed, with a special emphasis on the factors of human perception [6]. This not only greatly promoted the development of psychology but also provided a powerful theoretical support for later scholars to study psychology.

Domestic researchers also have some unique understanding of the cognitive learning model: Zeng, Hong, and others explored the cognitive learning model through a lot of analysis and argumentation to a large extent determines the quality of online learning outcomes [7]; Zhang, Tong, and others summarized the learning model by establishing scientific experiments. Good or bad has a significant impact on learning results [8]. Based on the information processing learning model, Gao, Zhongke, and others established an interactive cognitive complexity learning model and applied the model to teach design and proposed a teaching model with personal characteristics [9]. Lu et al. conducted a detailed analysis of the cognitive psychological process for users' information services [10]. It proposes a form of knowledge management based on cognitive psychology and analyzes the information service model in detail from the influencing factors such as perception and attention in human psychological process and the guiding role of these psychological processes on information service [11]. These achievements all explain how to use cognitive psychology to encourage users to effectively use information resources, avoiding the waste and idleness of extensive information resources that have evolved with time [12].

In general, both domestic and foreign researches on the design of neural network have already achieved certain results from the perspective of cognitive psychology. Relatively speaking, the two still play a leading role under the scope of theoretical research abroad. The research is more profound and has a theoretical basis for practice. In this paper, through the research of neural network design based on cognitive psychology, the psychological mechanism behind the design of neural network elements is explored to improve the efficiency of neural network element design and resource utilization. Cognitive mental model is based on fuzzy comprehensive evaluation [13]. Based on the traditional cognitive psychology model, an expanded cognitive psychology model is constructed. Based on controlling the untrue data through the repeated answer rate of the test questions and the vector recording method, the logical composition structure of the cognitive psychological model is designed. At the same time, the improved AHP and partial correlation analysis method are used to determine the weight of the factors of fuzzy comprehensive evaluation, and the unit cognitive evaluation model and the disciplinary comprehensive evaluation model are constructed, respectively.

\section{Cognitive Psychological Neural Network Model}

2.1. Cognitive Learning Psychological Impact Classification. The cognitive process itself is a mental process for the brain to process information (Figure 1). It refers to how to find and explain the world around you in your own way of understanding, create new knowledge and information through your own understanding and experience to solve difficult problems in life, seek out rules, and adjust and manipulate psychological processes in order to make information be obtained easier. The whole cognitive process includes four parts [14]:

(1) Perception, which is a collective term for feeling and perception, is actually a kind of reception of the information transmitted by the external world and the beginning of all cognitive processes. Information is transmitted to the human brain in the actual form of words, language, the human visual and auditory senses, and the signal of sensory information [15].

(2) Attention is to draw people's attention to it with a vivid image to focus on the ideology and achieve the role occupied by the human heart. Only when the ideology is optimized can people achieve the best state of perception and organize the sensory information in the brain.

(3) Memory is defined as a physiological phenomenon that stores and extracts information in the natural world. The memory process is to encode, store, and retrieve information. Coding is actually the acquisition of information, which is the first process of processing information, thus forming a memory representation. Storage is to keep the coded information. The extraction is to keep the stored information for recovery and reuse. 


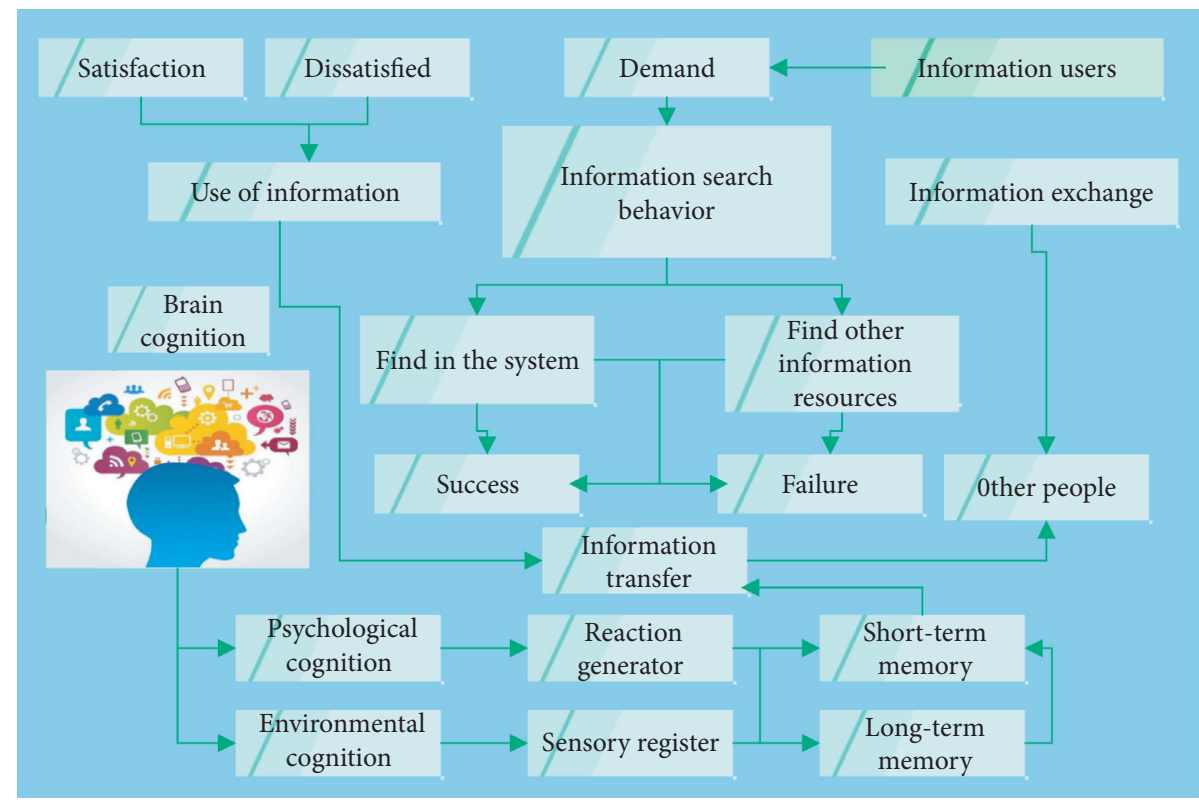

FIGURE 1: Cognitive learning psychological behavior.

(4) Use thinking and reasoning to solve problems. Thinking is also an internal cognitive process, which is to reprocess the internally stored long-term memory, that is, the so-called reorganization of longterm memory information, finely retell it, extract clues, compare information, and classify it. It is a deeper processing of long-term memory information [16].

Emotions are the driving force of all behaviors. It is precisely because of the triggering of emotions that the motivation to complete the behavior is generated. It is the guidance of emotions that enables the individual to maintain the completion of the established target behavior [17]. Therefore, it also plays a decisive role in information acquisition behavior. In the process of implementing information acquisition behavior, the difference in emotion directly affects the outcome of the behavior. Positive emotions directly stimulate the progress of the actors, so that the implementers of the behavior can continue to advance toward the goal of information acquisition. The information behavior is also more efficient, so that the behavior task can be performed more satisfactorily. However, under negative emotions, it will hinder information acquisition behavior, so that the behavior may not be completed, or it is difficult to achieve the desired effect during the entire information acquisition process.

From the perspective of information acquisition, although attitude is a good predictor, it is not an accurate indicator. Attitude will also elicit three responses. For example, I watched a movie that was vulgar (cognitive); disliked the movie (emotion); and unwilling to spend money to watch this bad movie (behavior). Sometimes, the information user is not interested in some information but still obtains this kind of information in a large amount; however, sometimes the information acquisition behavior is still closely followed by the attitude; for the information that matches the interest, the acquirer will spend more energy, and the information obtained is also more abundant and efficient.

2.2. Model Buildings. Human's understanding of the objective world often has a certain degree of ambiguity. The analysis of many problems cannot ignore the ambiguity attached to humans' view of the problem. Online learning evaluation cannot avoid the participation of human subjective consciousness activities, so it must be affected by human's own character, preferences, experience, knowledge, and technical level.

The neural network evaluation method obtains the weight of each node by training the sample set, thereby establishing a neural network evaluation model for online learning quality evaluation. Although this method avoids subjective factors in the process of obtaining weight, the entire process is a "dark box" operation, and the complete data processing process is difficult to be understood and accepted [18]. Fuzzy comprehensive evaluation analyzes complex objects from a hierarchical perspective and is not limited to linear models, which satisfy the requirements for evaluating complex objects. The processing of fuzzy comprehensive evaluation can be given by mathematical expressions, and the evaluation results are also expressed in the form of vectors, and the evaluation information is rich and clear at a glance. The evaluation level is shown in Table 1.

By establishing a fuzzy mapping relationship between the influencing factor set $E$ and the rating evaluation level $J$, a fuzzy matrix representation is established:

$$
P: E \longrightarrow G(J), e_{i} \longrightarrow P\left(e_{i}\right)=\left(h_{i 1}, h_{i 2}, \ldots, h_{i n}\right) \in G(J) .
$$


TABle 1: Evaluation level.

\begin{tabular}{lcc}
\hline Absolute score range & $\begin{array}{c}\text { Grade } \\
\text { indication }\end{array}$ & $\begin{array}{c}\text { Corresponding } \\
\text { comment }\end{array}$ \\
\hline $0 \leq x \leq 40$ & Difference & Very poor performance \\
$40 \leq x \leq 60$ & Poor & Poor performance \\
$60 \leq x \leq 70$ & Pass & Still need to work \\
$70 \leq x \leq 80$ & Medium & Average performance \\
$80 \leq x \leq 90$ & Good & Good performance \\
$90 \leq x \leq 100$ & Excellent & Excellent performance \\
\hline
\end{tabular}

After constructing the hierarchical fuzzy subset, the evaluation items are quantified from each factor $e_{i}(i=1,2 \ldots, n)$ one by one. The value of each element in the fuzzy relationship matrix represents the degree of membership of the evaluation index to the evaluation level, and the fuzzy relationship matrix $M$ is obtained:

$$
M=\left[\begin{array}{c}
M\left(e_{1}\right) \\
M\left(e_{2}\right) \\
\ldots \\
M\left(e_{n}\right)
\end{array}\right]=\left[\begin{array}{cccc}
h_{11} & h_{12} & \ldots & h_{1 \mathrm{~m}} \\
h_{21} & h_{22} & \ldots & h_{2 m} \\
\ldots & \ldots & \ldots & \ldots \\
h_{n 1} & h_{n 2} & \ldots & h_{n m}
\end{array}\right] .
$$

A suitable fuzzy operator combines fuzzy evaluation matrix $M$ and the corresponding weight vector $Y$ to obtain a comprehensive evaluation result.

$$
\begin{aligned}
F & =Y \circ M, \\
f_{j} & =\bigvee_{i=1}^{m}\left(y_{i} \wedge h_{i j}\right)(j=1,2, \ldots, n) .
\end{aligned}
$$

The result of fuzzy comprehensive evaluation is determined according to the evaluation result set. In the case of multiple comment levels, the conversion to point values is beneficial to the analysis. Usually there are maximum membership method and the weighted average method. The maximum membership degree is to select the largest evaluation result in the evaluation result vector as the final quantification of vague comments. If you need to compare the results vertically or horizontally, the result of the fuzzy evaluation vector is relatively rough. For this, the grade assignment method can give the average relative value $J$ of each grade according to the previously determined evaluation grade parameters and fuzzy operators $J=(40,50,60$, 70,80 , and 90). The weighted average method is used to calculate the comprehensive score, as shown in the following formula:

$$
A=\sum_{I=1}^{6} F_{i}^{*} J_{i}
$$

The traditional cognitive psychology model only obtains the evaluation value of the cognitive ability model obtained after learning a certain chapter through modelling [19]. To achieve the comprehensive evaluation value of the entire training activity, the traditional cognitive psychology model is used. Based on this, an expanded cognitive psychology model is proposed, namely, a comprehensive evaluation model. Its logical structure is shown in Figure 2.
The cognitive ability vector table is shown in Table 2 , and the vector $h_{i}=\left(h_{1}, h_{2}, h_{3}, h_{4}, h_{5}, h_{6}\right)$ is obtained. $h_{i} \in[-1.0$, 1.0], $1 \leq i \leq 6$, which means the correct rate of a certain cognitive ability, as shown in the following formula:

$$
m_{i}=\frac{B_{i}(1.0)}{B_{i}(1.0)+B_{i}(-1.0)} \text {. }
$$

Taking the comprehensive evaluation result as $q$ single factor evaluations in $J$ and setting the new weight distribution as $C$, the total fuzzy evaluation matrix $M$ is

$$
M=\left[\begin{array}{c}
C_{1} \\
C_{2} \\
\cdots \\
C_{m}
\end{array}\right]=\left[C_{i j}\right]_{m * n}
$$

2.3. Model Evaluations. The comprehensive evaluation system constructed in this paper is based on the unit evaluation model. That is, the unit cognitive evaluation value and the unit importance degree are vaguely synthesized and then fuzzy comprehensively integrated with the noncognitive indicators of the entire discipline, thereby evaluating the entire academic activity [20]. Among them, the importance of the unit and the weight of noncognitive indicators are determined by the improved analytic hierarchy process. This is because, according to the actual situation, different evaluation models use different weight determination methods according to the characteristics of the factors, so that the distribution of weight coefficients is more scientific and reliable. Through this evaluation mechanism, you can understand the actual situation of the cognitive level from different perspectives and try to reflect the actual training effect of learning as real and complete as possible.

Suppose that the operation of the convolutional layer can be expressed by PM, where $H$ represents the input, its dimension is $\beta^{*} M_{\mathrm{in}}{ }^{*} H_{\mathrm{in}}, M$ stands for the weight parameter, and the dimension of $M$ is $\beta^{*} M^{*} H$. Then replace the original weight $M$ parameter $C$ with a two-valued convolution kernel $C$ and a scale parameter $\beta$ whose dimension of $C$ is relative to $M$. Then there are

$$
\begin{aligned}
H^{*} M & \approx(H \oplus M) \beta, \\
F(C, \beta) & =\|M-\beta C\|^{*}\|H-\beta C\| .
\end{aligned}
$$

It is hoped that the optimization objective functions $J$ are as small as possible. In addition, the matrices $C$ and $M$ are converted into vectors, and the dimensions of $C$ and $M$ are $l^{*} n, n=\beta^{*} M^{*} H$.

$$
\begin{aligned}
m^{*}, h^{*} & =\arg \min F(C, \beta), \\
m^{*} & =\frac{1}{n}\left\|M_{i j}\right\|_{m^{*} n} .
\end{aligned}
$$

After comprehensively considering the calculation characteristics of each layer of the convolutional neural network and the performance characteristics of FPGA and software, a specific division was made to reduce the number 


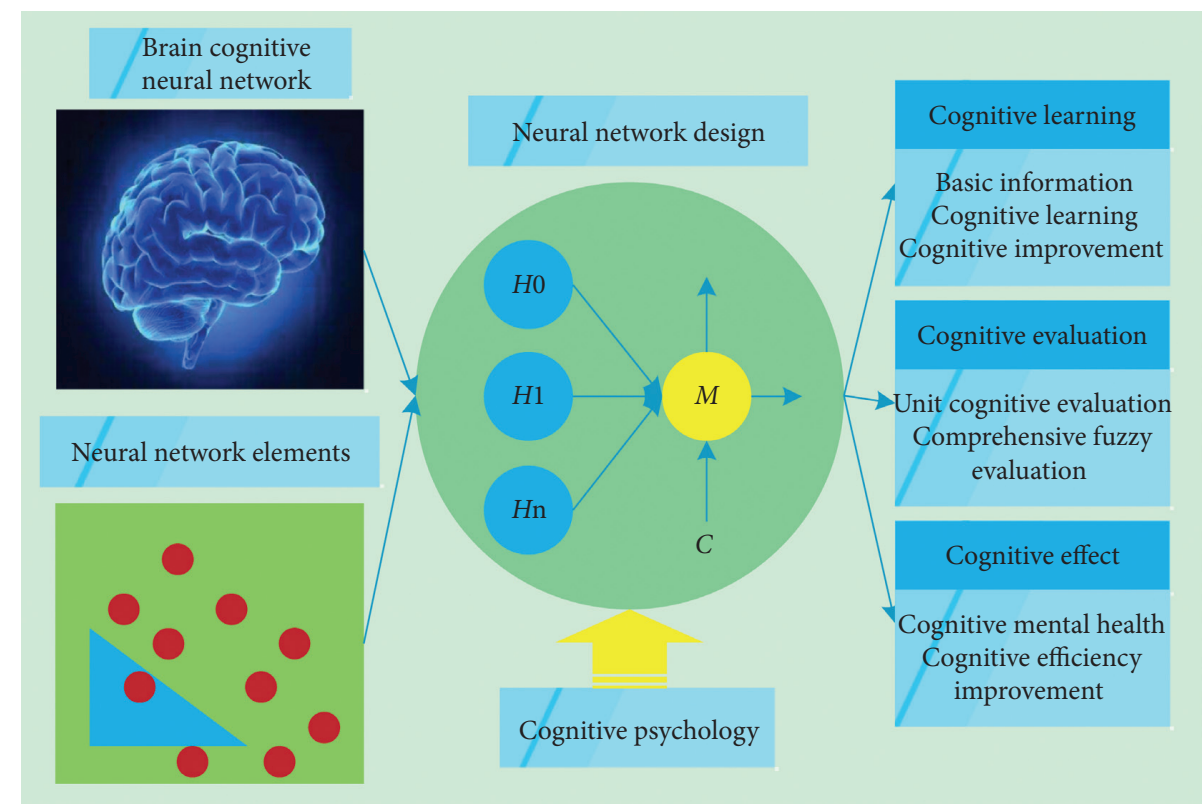

Figure 2: Cognitive psychological neural network.

TABLE 2: Cognitive vector table.

\begin{tabular}{lcccccc}
\hline Type & Remember & Understanding & Application & Analysis & Comprehensive & Evaluation \\
\hline $\mathrm{B}_{1}$ & 1.0 & 0.5 & -0.5 & 0 & 0.5 & 0.5 \\
$\mathrm{~B}_{2}$ & -0.5 & -0.5 & -1.0 & 0.5 & -0.5 \\
$\mathrm{~B}_{3}$ & -1 & 0 & 0.5 & -0.5 & -0.5 & 0.5 \\
$\mathrm{~B}_{4}$ & 0.5 & 1.0 & 0 & -1.0 & -0.5 & 1.0 \\
\hline
\end{tabular}

of parameters and the more calculation-intensive convolutional layer, pooling layer, and activation function. Put in FPGA part processing, the number of parameters is larger, and the fully connected layer with less calculation is put in software part processing [21]. This not only reduces the communication time between FPGA and software but also avoids spending a lot of FPGA logic resources to process many parameters of the fully connected layer. This approach makes the overall acceleration effect further improved. Figure 3 shows the logical structure of the improved evaluation model $[22,23]$.

\section{Results Analysis}

3.1. Cognitive Learning Psychological Classification Analysis. The survey data on the evaluation of the knowledge acquisition results are as follows (see Figure 4). After acquiring the information, the final evaluation of the information acquisition results must be carried out in order to truly identify and filter the absorbed information and solve the problem. According to the collected data, we found that the characteristics of information acquisition behavior embodied in this aspect are as follows:

(1) The awareness of information is gradually increasing. Most of them have realized that information occupies an extremely important position in our daily life and has a high degree of importance.
However, they are not cautious in their attitudes towards information, and they do not have the ability and attitude to identify the final information acquisition results.

(2) The final evaluation of information acquisition results is not high in cognitive satisfaction. After encountering difficulties in the implementation of acquisition behavior, many will generate self-doubt and question the ability to acquire, thus affecting the efficiency of the entire information acquisition behavior

Among information users, cognitive ability limits the final information acquisition behavior results. The lack of cognitive ability has caused college students to show a state of "heartlessness" towards information. Because of this "feeling of powerlessness," negative emotions such as anxiety and uncertainty are caused towards the information. Only when faced with a problem can it be said that it is forced to obtain it actively, resulting in a passive state model for information. Cognitive ability results are shown in Figure 5.

In the process of implementing information acquisition behavior, after information needs are generated, confusion, uncertainty, and anxiety may also occur, and the content of the information obtained may be related to its own cognitive process, as well as the accumulation of knowledge and experience. Violation, therefore, will have a negative impact on behavior. The cognitive behavior correction method is 


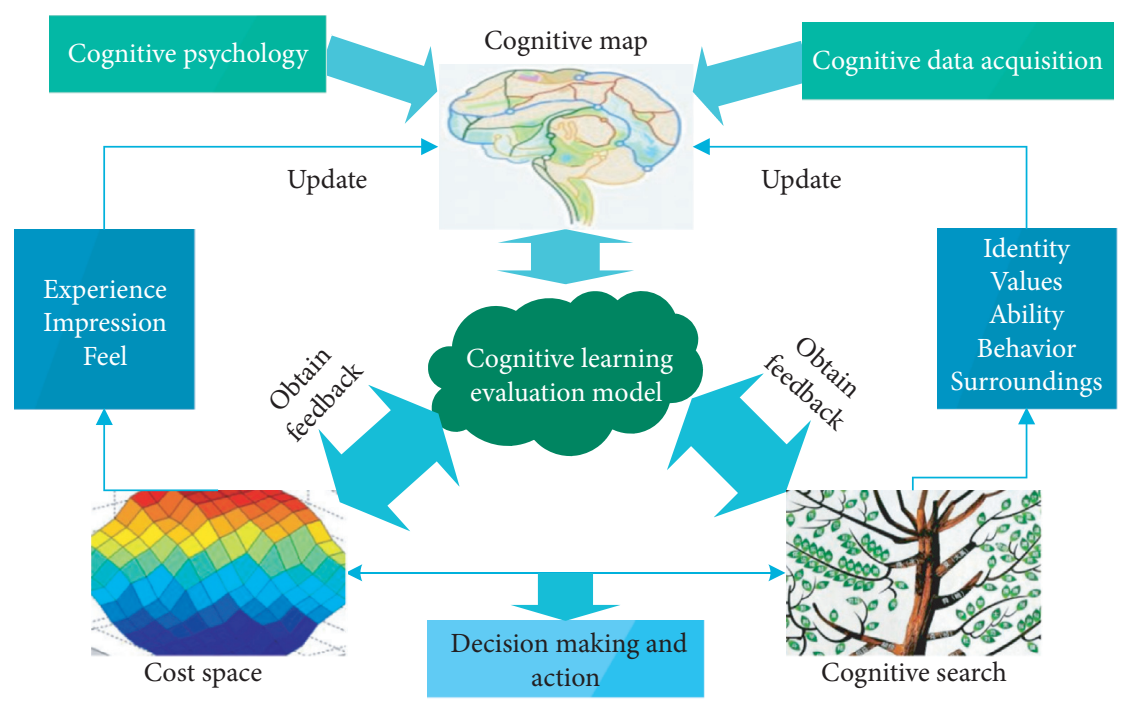

FIgure 3: Model evaluation logic.



Figure 4: Evaluation of psychological cognition.

that when information users encounter such obstacles in information acquisition behavior, they establish their own psychological construction model, change the past wrong consciousness and cognitive process, and consider their own cognitive abilities and cognitive style. They have the courage to improve negative attitudes, wrong reasoning to solve problems, and rigid cognitive style patterns. Under the situation that information acquisition behavior is affected by these negative and unfavourable psychological factors and cannot be performed normally, this rational emotional therapy can change the irrational psychology and overcome the severe anxiety caused by certain reactions caused by high 




$\square$ Quick change of mindset $\square$ Get nervous
$\square$ Transient anxiety $\quad \square$ Negative inverse mentality

Figure 5: Cognitive ability analysis.

emotional load emotions. Only by self-learning this kind of cognitive psychology treatment mode can we better implement information acquisition behavior.

3.2. Model Analyses. The results of the comprehensive evaluation of the subjects of the 80 students from the first and second phases of the training centre were selected, respectively. By comparison with their final online test score, the comparative analysis chart obtained is shown in Figure 6. Compared with the traditional training effect based on the final assessment score, fuzzy comprehensive evaluation is obviously more objective, which shows the true cognitive state and learning situation to a certain extent. Using only the final test score to evaluate the negation of the efforts and achievements of normal learning is not conducive to selfdevelopment. More importantly, the evaluation method adopted in this thesis has completely changed the situation that only focuses on the final exam results and ignores the usual learning process. In psychology, behavior is divided into consciousness and unconsciousness. A considerable part of our cognitive life involves unconsciousness. The difference between unconsciousness and consciousness is that the unconsciousness is an unnoticed behavior. Consciousness is the first time we face a certain thing or situation; we need to pass through the transmission of information and the coding of the brain and then to control our actions.

Through the statistical analysis of the comprehensive evaluation results of the two students, the fitting curve of frequency distribution and normal distribution shown in Figure 7 is obtained. It can be seen from the result distribution and normal curve fitting diagram shown in Figure 7 that the number of students with evaluation scores between 90 and 100 points accounted for $68.0 \%$, and the number of students with $80-90$ points accounted for $24.5 \%$. The number of people with 80 points is the largest, accounting for $32.7 \%$, the number of people with $60-70$ points is $10.2 \%$, the number of people with $40-60$ points is $56.0 \%$, and those with scores below 40 account for $27.0 \%$. Basically, it accords with the general student's grade distribution status. It can be seen from the fitted normal distribution graph that the student's academic performance is also concentrated around the median value, which relatively conforms to the normal distribution law of the student's performance. Based on the above analysis, the student's cognitive model based on fuzzy comprehensive evaluation has certain scientificality and feasibility and can play a good guiding role to meet the needs of the training platform.

To construct a missing data set, first set the missing rate of each input parameter to 0.2 to compare the prediction accuracy of different methods. The results show that the learning model based on RNN is better than the SVR method. When there are missing values, the advantages of the RNN method are more prominent. Because the influence of time on missing values is considered in the data training process, the prediction accuracy of the LSTM-Decay model proposed in this paper is higher than those of other LSTM benchmark methods. Figure 8 shows the prediction result of cognitive ability recognition rate in spatial and data sets. The result intuitively illustrates that the model can also effectively predict the time series changes for input data with missing values.

3.3. Correlation Analysis. In the process of implementing information acquisition behavior, the information service has established such a similar "cognitive map." In information navigation services, cognitive maps help users to obtain the information they need and produce the corresponding images in their minds. It builds a vivid and stereoscopic 3D image in people's minds and conveys the spatial relationship between the information and the information users in the form of visual cognition and visual representation, specific to the distribution of information, classification of categories, and access methods, greatly improving the efficiency of information acquisition behavior. Through the neural network element design method, improvement and prediction analysis is performed, and the results are shown in Figure 9. The improved method has the advantages of higher prediction accuracy, lower error rate, and stronger model adaptation. However, the execution time is relatively long and the model is complicated.

Because everyone's perception will be affected by psychological factors, perception will again process the sensed information to process the information, projecting selfconsciousness and meaning, and finally the acquired information has a personal style. For the same information source, the information obtained by everyone will be different. For example, the objects seen in the shape of the cloud will differ according to the information user. This is because the information users project their own knowledge and experience of the obtained information. Make a correct cognition and understanding of self, and, from self-concept, a dynamic psychological structure. It can initiate, explain, organize, transmit, and regulate inner and information acquisition behavior. The content of self-concept includes memory, cognitive style, cognitive ability, motivation, and even consciousness. To correctly understand self-concept and form self-schema should be learned; it can organize information about itself and manage and gradually enrich 


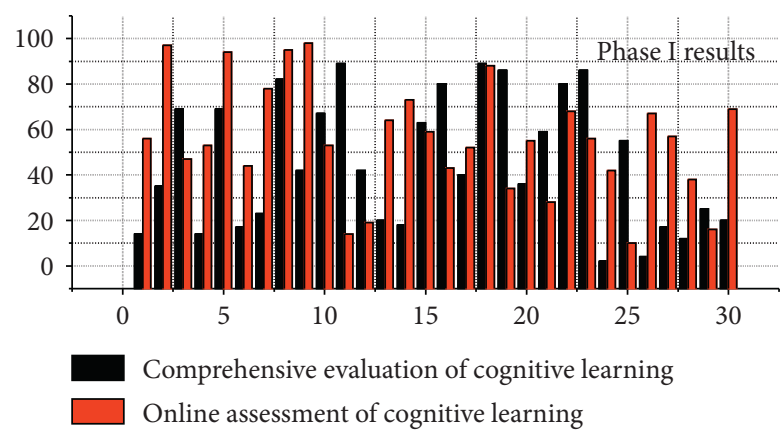

(a)

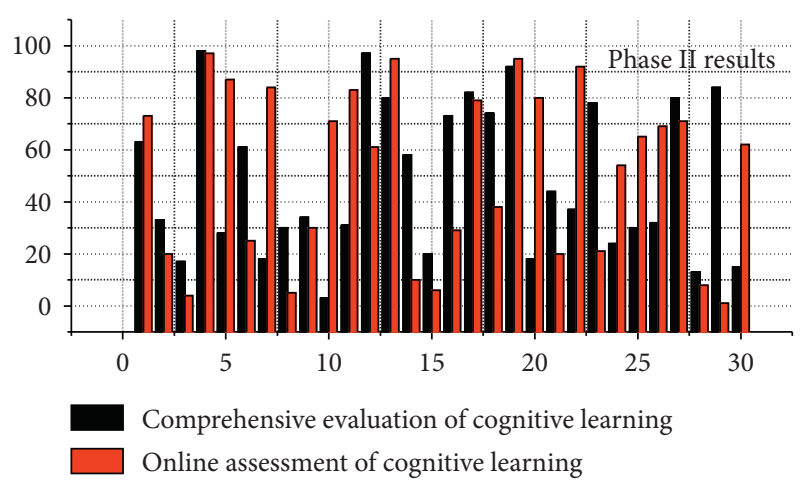

(b)

FIGURE 6: A comparison chart of the fuzzy comprehensive evaluation results of the first and second periods and the final examination results.

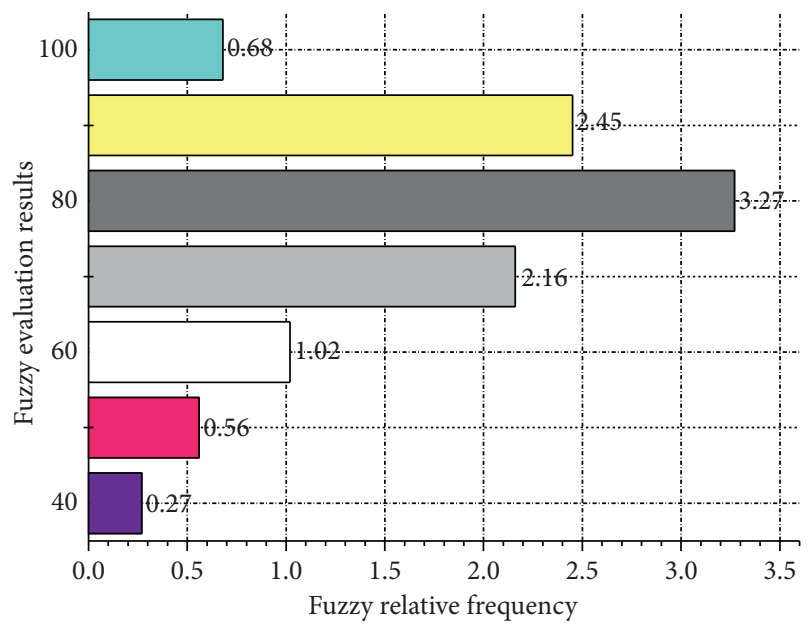

Figure 7: Comprehensive evaluation score distribution and normal curve fitting diagram.

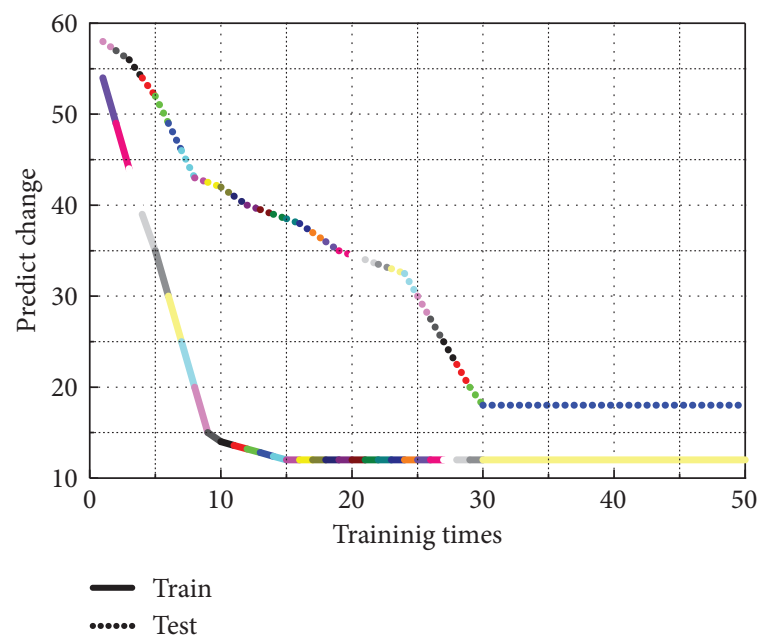

FIgURE 8: Training analysis results.

knowledge and experience, as well as affecting self-information consciousness and information processing process. Only from the psychological point of view of having a

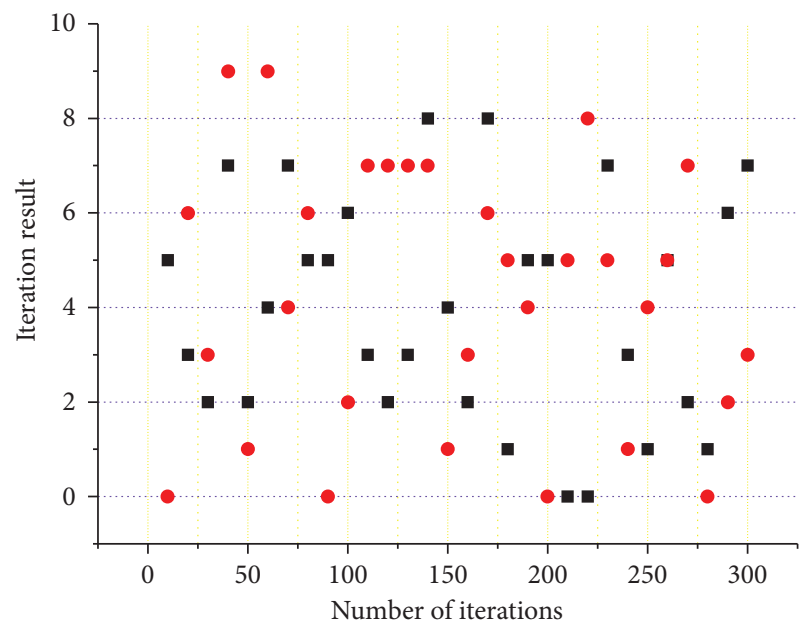

- Forecast result

- Real data

Figure 9: Comparison of prediction results and real data.

correct understanding and understanding of the self can the information acquisition behavior be clear, the information needs of the self be manifested, and the information that best meets the information need be found.

With the development of pedagogy and cognitive science theories, research has found that the factors that affect the development of cognitive levels have increased. It may not be comprehensive enough to evaluate students' cognitive levels from these aspects. Therefore, with the increase and improvement of evaluation indicators, the cognitive study model discussed in this article must be able to be adjusted in real time, because this functional expansion often requires changes from the underlying details such as modelling methods and database structure. This inevitably requires the suggestion of a more flexible and safe expansion mechanism, which can flexibly expand the models established in the past on the basis of ensuring effectiveness. Later in this area, we need to focus on research.

In the fuzzy comprehensive evaluation, the relative average value of the evaluation level is $35,45,55,65,75,85$, and 95 , respectively, which causes that even if all the evaluation 
indicators of a student are excellent, his cognitive ability has the highest score. It can only be 95. Similarly, if the evaluation indicators of the students are very low, the minimum score is also 40 points. This is a drawback of the algorithm, and further research is needed later.

\section{Conclusion}

To improve the efficiency of cognitive learning behavior, this paper proposes a cognitive mental model based on neural network design. Suggestions for subject psychology are as follows: (1) overcome negative emotions based on "cognitive correction"; (2) correctly understand the structure of selfknowledge experience; (3) develop strong conscious motivation based on expectations and self-realization. Suggestions based on the information service industry perspective are as follows: (1) establish a guiding information service based on cognitive psychology; (2) provide and create a good information service environment; (3) enhance information resource navigation services; (4) enhance the cognitive psychology of information service related personnel learning quality. Students are evaluated by building a cognitive student model based on fuzzy comprehensive evaluation. The expanded cognitive student model is divided into unit cognitive evaluation model and disciplinary comprehensive evaluation model according to different learning stages. Among them, this study proposes the use of improved AHP and partial correlation analysis for the determination of weight vectors in fuzzy comprehensive evaluation models, which greatly improves the persuasiveness and reliability of weight determination. This article combines the theoretical research related to cognitive psychology and neural network design. From the analysis of human cognitive process, it will explain two different interpretations of the human cognitive process: information processing theory, cognitive theory, and neural network design. Combining, pointing out its corresponding theoretical integration points, and proposing a relative application system have important value and significance for how to improve cognitive learning and mental health.

\section{Data Availability}

The data used to support the findings of this study are available from the corresponding author upon request.

\section{Conflicts of Interest}

The authors declare that they have no conflicts of interest reported in this paper.

\section{Acknowledgments}

The authors acknowledge Ministry of Science and Technology, "the Second Comprehensive Scientific Investigation on the Qinghai-Tibet Plateau," Topic on Life Geochemical Cycle and Environmental Health, under Project no. 2019QZKK0606; Department of Science and Technology of Qinghai Province, Key Laboratory of Medicinal Animal and Plant Resources of Qinghai-Tibetan Plateau in Qinghai
Province, Xining 810008, China, under Project no. 2020-ZJY40; and the Research on the Relationship between Psychological Resilience, Academic Emotion and Learning Engagement of Tibetan Middle School Students in Qinghai Province, under Project no. 20QJG46.

\section{References}

[1] W. Zhao, S. Du, and W. J. Emery, "Object-based convolutional neural network for high-resolution imagery classification," IEEE Journal of Selected Topics in Applied Earth Observations and Remote Sensing, vol. 10, no. 7, pp. 33863396, 2017.

[2] Q. Zhou, F. Chao, and C.-M. Lin, "A functional-link-based fuzzy brain emotional learning network for breast tumor classification and chaotic system synchronization," International Journal of Fuzzy Systems, vol. 20, no. 2, pp. 349-365, 2018.

[3] D. Bzdok and A. Meyer-Lindenberg, "Machine learning for precision psychiatry: opportunities and challenges," Biological Psychiatry: Cognitive Neuroscience and Neuroimaging, vol. 3, no. 3, pp. 223-230, 2018.

[4] G. Wen, Z. Hou, H. Li, D. Li, L. Jiang, and E. Xun, "Ensemble of deep neural networks with probability-based fusion for facial expression recognition," Cognitive Computation, vol. 9, no. 5, pp. 597-610, 2017.

[5] N. Kasabov, L. Zhou, M. G. Doborjeh, Z. G. Doborjeh, and J. Yang, "New algorithms for encoding, learning and classification of fMRI data in a spiking neural network architecture: a case on modeling and understanding of dynamic cognitive processes," IEEE Transactions on Cognitive and Developmental Systems, vol. 9, no. 4, pp. 293-303, 2017.

[6] B. Kanchanatawan, S. Thika, S. Sirivichayakul, A. F. Carvalho, M. Geffard, and M. Maes, "In schizophrenia, depression, anxiety, and physiosomatic symptoms are strongly related to psychotic symptoms and excitation, impairments in episodic memory, and increased production of neurotoxic tryptophan catabolites: a multivariate and machine learning study," Neurotoxicity Research, vol. 33, no. 3, pp. 641-655, 2018.

[7] H. Zeng, C. Yang, G. Dai, F. Qin, J. Zhang, and W. Kong, "EEG classification of driver mental states by deep learning," Cognitive Neurodynamics, vol. 12, no. 6, pp. 597-606, 2018.

[8] T. Zhang, W. Zheng, Z. Cui, Y. Zong, J. Yan, and K. Yan, “A deep neural network-driven feature learning method for multi-view facial expression recognition," IEEE Transactions on Multimedia, vol. 18, no. 12, pp. 2528-2536, 2016.

[9] Z. Gao, X. Wang, Y. Yang et al., "EEG-based spatio-temporal convolutional neural network for driver fatigue evaluation," IEEE Transactions on Neural Networks and Learning Systems, vol. 30, no. 9, pp. 2755-2763, 2019.

[10] H. Lu, Y. Li, M. Chen, H. Kim, and S. Serikawa, "Brain intelligence: go beyond artificial intelligence," Mobile Networks and Applications, vol. 23, no. 2, pp. 368-375, 2018.

[11] D. Durstewitz, G. Koppe, and A. Meyer-Lindenberg, "Deep neural networks in psychiatry," Molecular Psychiatry, vol. 24, no. 11, pp. 1583-1598, 2019.

[12] U. Hasson, S. A. Nastase, and A. Goldstein, "Direct fit to nature: an evolutionary perspective on biological and artificial neural networks," Neuron, vol. 105, no. 3, pp. 416-434, 2020.

[13] G. E. Morales-Martinez, E. O. Lopez-Ramirez, C. CastroCampos, M. G. Villarreal-Trevino, and C. J. Gonzales-Trujillo, "Cognitive analysis of meaning and acquired mental representations as an alternative measurement method technique 
to innovate E-assessment," European Journal of Educational Research, vol. 6, no. 4, pp. 455-465, 2017.

[14] M. Pratama, G. Zhang, M. J. Er, and S. Anavatt, "An incremental type-2 meta-cognitive extreme learning machine," IEEE Transactions on Cybernetics, vol. 47, no. 2, pp. 339-353, 2016.

[15] D. Harris and W.-C. Li, "Using Neural Networks to predict HFACS unsafe acts from the pre-conditions of unsafe acts," Ergonomics, vol. 62, no. 2, pp. 181-191, 2019.

[16] S. S. S. Kruthiventi, K. Ayush and R. Venkatesh Babu, Deepfix: a fully convolutional neural network for predicting human eye fixations," IEEE Transactions on Image Processing, vol. 26, no. 9, pp. 4446-4456, 2017.

[17] J. S. Bowers, "Parallel distributed processing theory in the age of deep networks," Trends in Cognitive Sciences, vol. 21, no. 12, pp. 950-961, 2017.

[18] J. C. Peterson, J. T. Abbott, and T. L. Griffiths, "Evaluating (and improving) the correspondence between deep neural networks and human representations," Cognitive Science, vol. 42, no. 8, pp. 2648-2669, 2018.

[19] D. C. Briggs and R. Circi, "Challenges to the use of artificial neural networks for diagnostic classifications with student test data," International Journal of Testing, vol. 17, no. 4, pp. 302-321, 2017.

[20] Y. Rizk, N. Hajj, N. Mitri, and M. Awad, "Deep belief networks and cortical algorithms: a comparative study for supervised classification," Applied Computing and Informatics, vol. 15, no. 2, pp. 81-93, 2019.

[21] L. Dye, N. B. Boyle, C. Champ, and C. Lawton, "The relationship between obesity and cognitive health and decline," Proceedings of the Nutrition Society, vol. 76, no. 4, pp. 443454, 2017.

[22] N. E. Anderson, V. R. Steele, J. M. Maurer et al., "Differentiating emotional processing and attention in psychopathy with functional neuroimaging," Cognitive, Affective, \& Behavioral Neuroscience, vol. 17, no. 3, pp. 491-515, 2017.

[23] J. A. Anderson, "Cognitive and psychological computation with neural models," IEEE Transactions on Systems, Man, and Cybernetics, vol. SMC-13, no. 5, pp. 799-815, 1983. 